\title{
Incidence of bacteraemia following endobronchial ultrasound-guided transbronchial needle aspiration
}

\author{
D.P. Steinfort*, D.F. Johnson" and L.B. Irving*
}

ABSTRACT: Few data exist concerning possible infectious complications associated with endobronchial ultrasound-guided transbronchial needle aspiration (EBUS-TBNA). The present prospective evaluation was undertaken in order to determine the incidence of bacteraemia and infectious complications associated with EBUS-TBNA.

Consecutive patients undergoing EBUS-TBNA for evaluation of mediastinal or hilar lymph node lesions were studied. Venesection was performed within $60 \mathrm{~s}$ of TBNA for aerobic and anaerobic blood culture. Sterile saline washing of TBNA needles was also performed. Patients with positive blood cultures were reviewed immediately, and all patients underwent clinical review within 1 week of EBUS-TBNA.

A total of 43 patients underwent EBUS-TBNA, with bacteraemia demonstrated in three (7\%). All bacterial isolates were typical oropharyngeal commensal organisms. The TBNA needle washing culture was positive in 15 (35\%) patients. None of the three bacteraemic patients had clinical features suggestive of infection, and no complications were seen among the cohort.

The incidence of bacteraemia following EBUS-TBNA is comparable to that following routine flexible bronchoscopy. Performance of TBNA does not appear to measurably increase the risk of bacteraemia over that associated with insertion of the bronchoscope into the airway. Contamination of the TBNA needle with oropharyngeal commensal bacteria is common; however, clinically significant infection following EBUS-TBNA appears rare.

\section{KEYWORDS: Complications, endobronchial ultrasound, infection, lung cancer}

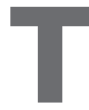

he recent introduction of endobronchial ultrasound-guided transbronchial needle aspiration (EBUS-TBNA) has revolutionised the evaluation of intrathoracic lymph nodes and other paratracheal structures. Since its description in 2004 [1], this minimally invasive bronchoscopic technique has achieved widespread popularity among respiratory physicians and thoracic surgeons, and, in centres where it is available, is now the procedure of choice for mediastinal staging of lung cancer. Its popularity is based, in part, on its excellent performance characteristics, but equally on its excellent safety profile. Its diagnostic accuracy is at least equivalent to that of mediastinoscopy and significantly greater for certain lymph node stations [2], and a recently published meta-analysis noted no important complications among $>1,500$ completed procedures [3].

Infective complications following conventional (Wang needle) TBNA have been reported, but prospective studies have suggested that bacteraemia following conventional TBNA is extremely rare [4]. Two recent reports have illustrated that
EBUS-TBNA may be associated with clinically significant infection, due to direct inoculation of oropharyngeal flora into mediastinal tissue by the TBNA needle $[5,6]$. A prospective study was conducted to determine the incidence of bacteraemia following EBUS-TBNA in order to inform clinicians regarding the risk of complicating infection. Culture of needle washings following TBNA was also performed in order to identify the microbial pathogens responsible for clinical infection following inoculation by TBNA. It was demonstrated for the first time that bacteraemia rates following EBUS-TBNA were consistent with rates reported following routine bronchoscopy. Culture of needle washings following TBNA identified organisms that routinely colonise the oropharanyx (15 out of $43 ; 35 \%$ ). No local infections or complications related to bacteraemia were identified.

\section{MATERIALS AND METHODS}

Approval was granted for the performance of the present study by the Melbourne Health Human

\section{AFFILIATIONS}

*Dept of Respiratory Medicine, Royal Melbourne Hospital, Parkville, and \#Dept of Infectious Diseases, Austin Hospital, Heidelberg, Australia.

\section{CORRESPONDENCE}

D.P. Steinfort

Dept of Respiratory Medicine, Level

1, Centre for Medical Research

Royal Melbourne Hospital

Parkville

Victoria 3050

Australia

E-mail: daniel.steinfort@mh.org.au

Received:

Sept 262009

Accepted after revision:

Nov 022009

First published online:

Nov 192009 
Research Ethics Committee (Parkville, Australia). All of the patients provided written informed consent.

All patients undergoing EBUS-TBNA for evaluation of mediastinal lesions were considered for inclusion in the present study. The following exclusion criteria were applied: current febrile illness, antibiotic therapy within the previous fortnight, current respiratory infection, and indication for prophylactic antibiotics.

EBUS-TBNA was performed under conscious sedation using a dedicated linear array bronchoscope (BF-UC180F-OL8; Olympus, Tokyo, Japan) by a single operator (D.P. Steinfort). Topical anaesthesia using $2 \%$ lidocaine was introduced via the working channel of the linear array bronchoscope. A single-use TBNA needle (NA-201SX-4022; Olympus) was used for each patient. In order to ensure assessment of bacteraemia that pertained specifically to EBUS-TBNA, TBNA was performed as the first diagnostic procedure, and blood cultures were drawn prior to any further diagnostic procedures being performed.

Venepuncture of an antecubital vein was performed within $60 \mathrm{~s}$ of completion of the final TBNA. A total of $20 \mathrm{~mL}$ blood was drawn and then divided equally between both aerobic (BD BACTEC Plus Aerobic/F 442192; Becton Dickinson, Hunt Valley, MD, USA) and anaerobic (BD BACTEC Plus Anaerobic/F 442193; Becton Dickinson) culture bottles. Specimens were incubated in an instrumented blood culture system (BD BACTEC 9240; Becton Dickinson) at $35^{\circ} \mathrm{C}$. Positive blood culture vials were processed according to the manufacturer's instructions.

Following completion of the procedure, $10 \mathrm{~mL}$ sterile normal saline was washed through the TBNA needle lumen into a sterile container and sent for microbial culture. Following centrifugation, material was inoculated on to both horse blood agar and chocolate agar and incubated under $\mathrm{CO}_{2}$ for $48 \mathrm{~h}$ at $35^{\circ} \mathrm{C}$. Patients whose blood cultures were positive were immediately contacted by telephone in order to review any symptoms that might suggest clinical infection. All of the patients underwent detailed clinical review at the time of the scheduled postprocedure review at 3-7 days following EBUS-TBNA.

\section{Statistics}

Comparison between patient groups was made using Fisher's exact test. This test, as well as the confidence interval (CI), was calculated using online software [7].

\section{RESULTS}

A total of 45 consecutive patients undergoing EBUS-TBNA between March 19 and August 21, 2009 consented to involvement in the present study. Two patients met exclusion criteria (one required prophylactic antibiotics and one had a concurrent febrile illness); therefore 43 patients had samples taken for analysis. The clinical indication for performance of EBUS-TBNA, as well as the final diagnosis, is recorded in table 1.

EBUS-TBNA was performed for evaluation of mediastinal lesions at a number of lymph node stations (table 2). The median \pm SD lesion size was $2.6 \pm 0.9 \mathrm{~cm}$ (range $0.9-3.5 \mathrm{~cm}$ ). The median number of needle passes performed prior to venesection was 2.3 (range 1-4). Sufficient material for pathological analysis was obtained in 41 (95\%) patients, and demonstrated a definitive diagnosis in 33, with TBNA results from eight patients demonstrating normal lymphoid tissue.

Peripheral blood culture samples taken within $60 \mathrm{~s}$ of TBNA were positive in three $(7.0 \% ; 95 \%$ CI $1.7-19.3 \%)$ patients. All of the organisms identified typically colonise the oropharnyx. The bacteria isolated from and clinical features of these three patients are recorded in table 3 . No significant relationship

TABLE 1 Indication for performance of endobronchial ultrasound-guided transbronchial needle aspiration and final diagnoses

Indication

Staging of known NSCLC

Mediastinal evaluation of suspected locally advanced NSCLC

Suspected sarcoidosis
Isolated mediastinal/hilar lymphadenopathy

Final diagnosis

Patients $n$

$\begin{array}{ll}\text { Adenocarcinoma } & 6 \\ \text { Squamous cell carcinoma } & 3 \\ \text { NSCLC } & 2 \\ \text { Large cell carcinoma } & 2 \\ \text { Sarcoid granulomas } & 1 \\ \text { Normal lymph node } & 6 \\ \text { Small cell lung carcinoma } & 4 \\ \text { Squamous cell carcinoma } & 3 \\ \text { Adenocarcinoma } & 1 \\ \text { Granulomatous inflammation } & 1 \\ \text { Normal lymph node } & 2 \\ \text { Sarcoidosis } & 5 \\ \text { Normal lymph node } & 1 \\ \text { Hodgkin's disease } & 2 \\ \text { Breast carcinoma metastases } & 2 \\ \text { Metastatic melanoma } & 1 \\ \text { Carcinoid tumour } & 1\end{array}$

NSCLC: nonsmall cell lung cancer 


\begin{tabular}{|c|c|}
\hline Lymph node station ${ }^{\#}$ & Patients $n$ \\
\hline 7 & 21 \\
\hline $4 R / 4 L$ & 13 \\
\hline 10R/L & 7 \\
\hline $2 R$ & 1 \\
\hline 1L & 1 \\
\hline
\end{tabular}

was seen between presence of bacteraemia and lesion size, number of needle aspirations performed or underlying pathology. Bacteraemia was even noted for one patient, undergoing EBUS-TBNA for evaluation of suspected mediastinal recurrence of breast carcinoma, from whom inadequate samples were obtained. None of the three bacteraemic patients showed clinical features suggestive of infection, and no complications were seen among the present cohort.

Culture of needle washings following TBNA was negative in $28(65 \%)$ patients. Cultures were positive in $15(35 \%)$ patients, with growth of multiple anaerobic and aerobic organisms, typical of upper respiratory tract flora, in 14 patients and a pure growth of Streptococcus mitis in one patient. Needle washing culture was negative in all three patients in whom bacteraemia was identified.

\section{DISCUSSION}

This is the first report to describe the frequency of bacteraemia following EBUS-TBNA, which appears comparable to the rate of bacteraemia associated with routine flexible bronchoscopy. A bacteraemia rate of $7 \%$ was recorded among patients undergoing EBUS-TBNA for evaluation of mediastinal and hilar lesions, although, importantly, none of the present patients experienced any clinically significant infective complications. The risk of bacteraemia did not appear to depend upon lesion size or the underlying pathology being sampled. It is also interesting to observe that bacteraemia may even occur despite an inadequate sample being retrieved by the procedure. The exact cause of bacteraemia following EBUS-TBNA remains unclear.

The rate of bacteraemia following routine flexible bronchoscopy varies between reports and ranges 0-6\% [9-13]. YigLA et al. [10] previously observed bacteraemia in $6 \%$ of patients undergoing bronchoscopy, comparable to the present observed bacteraemia rate, and noted no association between bacteraemia and performance of procedures (e.g. brushings and biopsy); they suggested that bacteraemia may result from bacterial mucosal penetration above the vocal cords, or, alternatively, due to bronchial mucosal trauma following introduction of the bronchoscope. This is supported by the observation, in both human and animal studies, that bacteraemia may be seen in $>30 \%$ of patients undergoing rigid bronchoscopy, in whom mucosal trauma is significantly greater than that seen for flexible bronchoscopy [14, 15].

It is of interest to observe that bacterial culture of the TBNA needle washings was negative in all three patients in whom bacteraemia was demonstrated. This may be a result of the reduced sensitivity of the agar culture method used for the needle washing compared with the BACTEC system used for detecting bacteraemia. It is also possible that the bacteraemia in the present patients was a result of introduction of the bronchoscope per se, and that performance of TBNA did not measurably increase the risk of bacteraemia. This is also consistent with the observation that, although infections at the site of needle puncture following conventional TBNA have been reported [16], the only study to examine bacteraemia rates following conventional TBNA observed no bacteraemia in 50 procedures [4]. In comparison to a routine bronchoscope (through which conventional TBNA is performed), the EBUSTBNA bronchoscope is larger and less manoeuvrable and has poorer video optics (including a $30^{\circ}$ viewing camera). This increases the likelihood of pharyngeal and glottic contact during introduction of the bronchoscope through the upper airway and may explain the higher bacteraemia rate observed here in comparison to those undergoing conventional TBNA [4].

Similarly, bacteraemia rates following upper gastrointestinal endoscopic ultrasound (EUS) are similar whether or not fine needle aspiration (FNA) is performed [17]. The observed incidence of bacteraemia following EUS is equal to that seen in routine gastroscopy $(6 \%)$ [18, 19], and, for both procedures, biopsy or other endoscopic operation does not appear to increase the likelihood of bacteraemia $[18,19]$.

EPSTEIN et al. [16] previously reported polymicrobial contamination of conventional TBNA needle contents in all patients examined. The present findings also indicate that contamination of the TBNA needle by oropharyngeal flora is common. It can also be observed that the majority of reports describing

\section{TABLE 3 Clinical features of patients with confirmed bacteraemia following transbronchial needle aspiration (TBNA)}

\begin{tabular}{|c|c|c|c|c|c|c|c|c|}
\hline \multirow[t]{2}{*}{ Patient No. } & \multirow[t]{2}{*}{ Sex } & \multirow[t]{2}{*}{ Age yrs } & \multicolumn{2}{|c|}{ Lesion } & \multirow[t]{2}{*}{ TBNA procedures $n$} & \multirow[t]{2}{*}{ Final diagnosis } & \multirow{2}{*}{ Bacterial isolate } & \multirow{2}{*}{$\begin{array}{c}\text { Clinically significant } \\
\text { infection }\end{array}$} \\
\hline & & & Location ${ }^{\#}$ & Size cm & & & & \\
\hline 1 & $\mathrm{~F}$ & 62 & 7 & 2.4 & 2 & Insufficient material" & Actinomyces spp. & No \\
\hline 2 & M & 77 & $10 \mathrm{~L}$ & 3.1 & 1 & Metastatic melanoma & Streptococcus salivarius & No \\
\hline 3 & M & 26 & 7 & 1.9 & 2 & Granulomatous inflammation & Streptococcus mitis & No \\
\hline
\end{tabular}

F: female; M: male. ${ }^{\#}$ : Mountain and Dresler lymph node station classification [8]; ": confirmed metastatic adenocarcinoma of breast by subsequent surgical biopsy. 
clinically significant infection following either TBNA or EUSFNA describe infection of relatively avascular tissue $[5,6,16$, $20,21]$. During insertion of the bronchoscope through the oropharynx, suctioning is very difficult to avoid while establishing a view of the larynx. Contamination of the bronchoscope channel lumen should be assumed for all EBUS-TBNA procedures. Although insertion of the bronchoscope through an endotracheal tube (under general anaesthetic) may avoid this, intubation itself is associated with a bacteraemia rate of $>10 \%$ [22], and the very low observed incidence of infection complicating EBUS-TBNA should not influence sedation practice.

Guidelines from the American Heart Association for the prevention of infective endocarditis acknowledge that respiratory tract procedures may cause transient bacteraemia with a wide array of microorganisms, but that the risk of antibioticassociated adverse events exceeds the benefit, if any, from prophylactic antibiotic therapy [23]. The present findings suggest that, given a comparable bacteraemia rate to routine flexible bronchoscopy, recommendations for use of prophylactic antibiotics for the prevention of infective endocarditis in patients undergoing EBUS-TBNA should not differ from those for routine bronchoscopy. Current guidelines recommend antibiotic prophylaxis for bronchoscopic procedures only in patients with cardiac conditions associated with the highest risk of adverse outcome from endocarditis [23].

Some limitations of the present study can be identified. Blood cultures were taken at only one time-point. However, rates of bacteraemia following dental procedures are highest immediately after the procedure, and fall dramatically after just 2 $5 \mathrm{~min}$ [24]. Therefore, the likelihood that bacteraemic events occurring after venesection were missed seems very low. Patients with fever were excluded, and no patients were immunocompromised at the time of EBUS-TBNA. Such patients may be at higher risk of bacteraemia or infective complications. Finally, culture of needle washings was performed by inoculation on to agar plates. The sensitivity of this method is $<100 \%$, and it is likely that the findings underestimate the true rate of needle contamination by oropharyngeal bacteria.

The present study cannot inform comment on the exact risk of local infections. Although bacterial contamination of the TBNA needle was demonstrated in $35 \%$ of patients, none developed evidence of clinically significant infection. Oropharyngeal commensal bacterial contamination of the TBNA needle is almost certainly a common event in EBUS-TBNA. Despite this, only two reports describe clinically significant infections complicating EBUS-TBNA [5, 6], suggesting local infection at the site of TBNA is either extremely rare or potentially underrecognised and/or under-reported.

Given the assumed needle contamination, we believe patient factors influence the risk of local infection during EBUS-TBNA more than do procedural factors. Future larger prospective studies are required in order to determine which lesions pose the greatest risk of local infection. Further evaluation of the cause of bacteraemia in EBUS-TBNA is also warranted, comparing bacteraemia rates following TBNA with rates following bronchoscope insertion, prior to performance of TBNA.
In conclusion, a low rate of bacteraemia was observed following performance of EBUS-TBNA, comparable to that previously reported following routine flexible bronchoscopy. Bacteraemia appears likely to be due to insertion of the bronchscope itself rather than to performance of TBNA. Contamination of the TBNA needle by oropharyngeal flora is common, and may predispose patients to clinically significant infection at the site of TBNA puncture, although this appears rare.

\section{STATEMENT OF INTEREST}

A statement of interest for D.P. Steinfort can be found at www.erj. ersjournals.com $/ \mathrm{misc} /$ statements.dtl

\section{REFERENCES}

1 Yasufuku K, Chiyo M, Sekine Y, et al. Real-time endobronchial ultrasound-guided transbronchial needle aspiration of mediastinal and hilar lymph nodes. Chest 2004; 126: 122-128.

2 Ernst A, Anantham D, Eberhardt R, et al. Diagnosis of mediastinal adenopathy-real-time endobronchial ultrasound guided needle aspiration versus mediastinoscopy. J Thorac Oncol 2008; 3: 577-582.

3 Varela-Lema L, Fernandez-Villar A, Ruano-Ravina A. Effectiveness and safety of endobronchial ultrasound-transbronchial needle aspiration: a systematic review. Eur Respir J 2009; 33: 1156-1164.

4 Witte MC, Opal SM, Gilbert JG, et al. Incidence of fever and bacteremia following transbronchial needle aspiration. Chest 1986; 89: 85-87.

5 Haas AR. Infectious complications from full extension endobronchial ultrasound transbronchial needle aspiration. Eur Respir J 2009; 33: 935-938.

6 Steinfort DP, Johnson DF, Irving LB. Infective complications from endobronchial ultrasound-transbronchial needle aspiration. Eur Respir J 2009; 34: 524-525.

7 GraphPad Software. QuickCalcs. www.graphpad.com/quickcalcs Date last updated: 2005. Date last accessed: September 25, 2009.

8 Mountain CF, Dresler CM. Regional lymph node classification for lung cancer staging. Chest 1997; 111: 1718-1723.

9 Kane RC, Cohen MH, Fossieck BE Jr, et al. Absence of bacteremia after fiberoptic bronchoscopy. Am Rev Respir Dis 1975; 111: 102-104.

10 Yigla M, Oren I, Bentur L, et al. Incidence of bacteraemia following fibreoptic bronchoscopy. Eur Respir J 1999; 14: 789-791.

11 Pedro-Botet ML, Ruiz J, Sabria $\mathrm{M}$, et al. Bacteriemia tras fibrobroncoscopia. Estudio prospectivo [Bacteraemia after fibrobronchoscopy. Prospective study]. Enferm Infecc Microbiol Clin 1991; 9: 159-161.

12 Durschmied H, Wilde J, Knoll P. Transitorische Bakteriämien in bronchologic Biopsie-Verfahren [Transitory bacteraemia in bronchological biopsy procedures]. Pneumologie 1990; 44: Suppl. 1, 208-209.

13 Haynes J, Greenstone MA. Fibreoptic bronchoscopy and the use of antibiotic prophylaxis. BMJ (Clin Res Ed) 1987; 294: 1199.

14 Burman SO. Bronchoscopy and bacteriemia. J Thorac Cardiovasc Surg 1960; 40: 635-639.

15 Nayci A, Atis S, Talas DU, et al. Rigid bronchoscopy induces bacterial translocation: an experimental study in rats. Eur Respir J 2003; 21: 749-752.

16 Epstein SK, Winslow CJ, Brecher SM, et al. Polymicrobial bacterial pericarditis after transbronchial needle aspiration. Case report with an investigation on the risk of bacterial contamination during fiberoptic bronchoscopy. Am Rev Respir Dis 1992; 146: 523-525.

17 Janssen J, Konig K, Knop-Hammad V, et al. Frequency of bacteremia after linear EUS of the upper GI tract with and without FNA. Gastrointest Endosc 2004; 59: 339-344.

18 Norfleet RG, Mitchell PD, Mulholland DD, et al. Does bacteremia follow upper gastrointestinal endoscopy? Am J Gastroenterol 1981; 76: $420-422$ 
19 Levy MJ, Norton ID, Wiersema MJ, et al. Prospective risk assessment of bacteremia and other infectious complications in patients undergoing EUS-guided FNA. Gastrointest Endosc 2003; 57: 672-678.

20 Aerts JG, Kloover J, Los J, et al. EUS-FNA of enlarged necrotic lymph nodes may cause infectious mediastinitis. J Thorac Oncol 2008; 3: 1191-1193.

21 Annema JT, Veselic M, Versteegh MI, et al. Mediastinitis caused by EUS-FNA of a bronchogenic cyst. Endoscopy 2003; 35: 791-793.

22 Valdes C, Tomas I, Alvarez M, et al. The incidence of bacteraemia associated with tracheal intubation. Anaesthesia 2008; 63: 588-592.
23 Wilson W, Taubert KA, Gewitz M, et al. Prevention of infective endocarditis: guidelines from the American Heart Association: a guideline from the American Heart Association Rheumatic Fever, Endocarditis, and Kawasaki Disease Committee, Council on Cardiovascular Disease in the Young, and the Council on Clinical Cardiology, Council on Cardiovascular Surgery and Anesthesia, and the Quality of Care and Outcomes Research Interdisciplinary Working Group. Circulation 2007; 116: 1736-1754.

24 Diz Dios P, Tomas Carmona I, Limeres Posse J, et al. Comparative efficacies of amoxicillin, clindamycin, and moxifloxacin in prevention of bacteremia following dental extractions. Antimicrob Agents Chemother 2006; 50: 2996-3002. 